\title{
Shifting Development in Mid-Childhood: The Influence of Between-Task Interference
}

\author{
Lucy Cragg $^{1}$, Kate Nation ${ }^{2}$ \\ ${ }^{1}$ Brain and Body Centre, University of Nottingham, UK. \\ ${ }^{2}$ Department of Experimental Psychology, University of Oxford, UK.
}

\section{This is the author's post-print of the article. The definitive version is published in Developmental Psychology, Volume 45, Issue 5, 2009. This can be accessed at http://doi.apa.org/getdoi.cfm?doi=10.1037/a0015360. This article may not exactly replicate the final version published in the APA Journal. It is not the copy of record.}

\begin{abstract}
Performance on the task-switching paradigm is greatly affected by the amount of conflict between tasks. Compared to adults, children appear to be particularly influenced by this conflict, suggesting that the ability to resolve interference between tasks improves with age. We used the task-switching paradigm to investigate how this ability develops in mid-childhood. Experiment 1 compared 5- to 8year-olds' and 9- to 11-year-olds' ability to switch between decisions about the colour of an object and its shape. The 5- to 8-year-olds were slower to switch task and experienced more interference from the irrelevant task than the 9-to 11-year-olds, suggesting a developmental improvement in resolving conflict between tasks during mid-childhood. Experiment 2 explored this further, examining the influence of stimulus and response interference at different ages. This was done by separating the colour and shape dimensions of the stimulus and reducing overlap between responses. The results supported the development of conflict resolution in task-switching during mid-childhood. They also revealed that a complex interplay of factors, including the tasks used and previous experience with the task, affected children's shifting performance.
\end{abstract}

Keywords: executive function, task-switching, development, congruence ${ }^{1}$

1 Address for correspondence:

Lucy Cragg

School of Psychology

University of Nottingham

University Park

NOTTINGHAM. NG7 2RD, UK

Tel: +441158467926

Fax: +441159515324

Email: lucy.cragg@,nottingham.ac.uk 


\section{Introduction}

Selecting the appropriate response for a given situation requires the correct configuration of mental resources, or 'task-set' to be in place before the task can be performed. In order to respond to changes in the environment it is necessary to be able to flexibly shift between these different task-sets. This is particularly important when the same stimulus requires different responses depending on the context e.g. answering a call on your cellphone or cutting it off depending on whether you are at home or in a meeting. The task-switching paradigm models such a situation. Participants must switch between making decisions about one of two dimensions of the same stimuli, such as its colour and shape, on a trial-by-trial basis. The stimuli remain the same across tasks; however the participant is required to switch their attention between these dimensions depending on the task being performed.

Switching to a new task results in a cost in performance. More errors are made, and responses are typically slower, when a new task is performed compared to when a task is repeated. Two different types of switching costs can be identified. The first are general, or mixing costs, which reflect the difference in RT between trials in a block in which just one task is performed (e.g. responding to the colour of an object) compared to trials in a mixed block in which two tasks are switched between (e.g. colour and shape decisions about an object). They are believed to reflect the difficulty in maintaining and selecting among two or more different potential response sets (Reimers \& Maylor, 2005). The second type, typically referred to as local or specific switch costs, represent the difficulty in reconfiguring a new task and response set. They are calculated by comparing the difference in RT between switch (e.g. a colour trial preceded by a shape trial) and non-switch trials (e.g. a colour trial preceded by a colour trial) within a mixed block.

\section{The task-switching paradigm as a measure of shifting in children}

There is a large literature concerning taskswitching in adults (see Monsell (2003) for a review), yet little is known about how the ability to shift develops beyond preschool years. Although lifespan development studies using the task-switching paradigm have shown larger performance costs in school-age children than in adults (Cepeda, Kramer, \& Gonzalez de Sather, 2001; Kray, Eber, \& Lindenberger, 2004; Reimers \& Maylor, 2005), it is not clear which aspects of performance improve during childhood. Kray, Eber and Lindenberger (2004) compared the switching ability of 8- to 10-year-old children, young adults and older adults and showed that the children had larger mixing costs than the young adults, suggesting a difficulty in maintaining and manipulating two task-sets in working memory. There was no difference in switch costs however. Reimers and Maylor (2005) also showed a developmental improvement in mixing costs, but not in switch costs, between 10 and 18 years of age. In contrast, Cepeda, Kramer and Gonzalez de Sather (2001) found that children and older adults showed significantly longer switch costs than the young adults, even when baseline speed was controlled. This difference in findings may be because Cepeda et al. included younger ( 7 to 9 years) and older (71 to 82 years) participants than Reimers and Maylor. These lifespan studies indicate that some aspects of performance on the taskswitching paradigm do improve with age. Potentially however, comparing across such large age-ranges, where large differences in performance would be expected, may have masked smaller developmental changes in switch costs within childhood.

Studies investigating task-switching changes within childhood and adolescence, rather than across the whole lifespan, have been more successful at demonstrating an improvement in shifting during childhood. Cohen, Bixenman, Meiran and Diamond (2001, as cited in Diamond (2002)) presented 5- to 11-year-olds and adults with a spatial version of the task-switching paradigm. Accuracy rather than RT was used as the dependent variable but a difference in error rate was shown between pure blocks and mixed blocks (mixing cost) and between switch and non-switch trials (switch cost). Furthermore, the difference in error rate decreased with age. Huizinga, Dolan and van der Molen (2006) also demonstrated a developmental improvement in the ability to switch between tasks during mid-childhood. Using RT as the dependent variable, they 
found a reduction in switch costs between the ages of 7 and 15 years on three versions of the task-switching paradigm. These studies give a better indication of the development of taskswitching in childhood, yet they do not explore what might be underlying these developmental changes. Research using the task-switching paradigm in adults has shown that a number of different processes can contribute to the switch cost. These include endogenous processes relating to preparation for the upcoming task (Rogers \& Monsell, 1995; Meiran, 1996), as well as interference and priming effects elicted by the stimuli themselves (Allport, Styles, \& Hsieh, 1994, Allport \& Wylie, 2000; Koch \& Allport, 2006; Waszak, Hommel, \& Allport, 2003; Wylie \& Allport, 2000). In this paper, we attempt to replicate the findings of Cohen et al. (2001) and Huizinga et al. (2006) and show a developmental improvement in taskswitching during mid-childhood. Furthermore, we investigate some of the factors that may affect children's performance, particularly interference effects, and see if these can account for developmental differences in performance.

\section{Processes contributing to the switch cost}

Part of the switch cost is thought to reflect the time taken for an endogenous control system to actively reconfigure the task-set (Meiran, 1996; Rogers \& Monsell, 1995). Evidence for this comes from the fact that if participants are given longer to prepare for the upcoming task, manipulated by extending the cue-target interval, then the switch cost is reduced (Meiran, 1996; Meiran, Chorev, \& Sapir, 2000; Rogers \& Monsell, 1995). Cepeda et al. (2001) found a reduced switch cost in children when the interval between the task cue and the target was increased, suggesting that children also reconfigure the upcoming task-set in advance, when possible.

The switch cost is also influenced by exogenous processes related to interference and priming of the stimuli and responses used for the tasks. For example, there is likely to be persisting interference from the competing stimulus-response (S-R) mappings that were relevant on the previous trial (Allport et al., 1994). Increasing the time between a response and the subsequent trial allows the activation from the previous task to dissipate, thus reducing the switch cost (Allport et al., 1994; Cepeda, Kramer, \& Gonzalez de Sather, 2001).
In addition to this persisting interference, the competing task can also be cued by the presentation of stimuli previously associated with this task (Allport \& Wylie, 2000; Koch \& Allport, 2006; Waszaket al., 2003; Wylie \& Allport, 2000).

Another factor that affects task-switching performance is the congruence of the stimuli. One of the main features of the task-switching paradigm is that the stimuli and responses are bivalent. In other words, the same stimuli and responses are used for both tasks. Attention has to be switched between two dimensions of the same stimulus, such as the colour and shape, and the same two response keys are then used to make decisions on both of these dimensions, creating conflict between the two tasks. Overlapping stimulus-response mappings creates some trials on which responses are congruent, where both tasks indicate the same response, and some that are incongruent, where conflicting responses are involved. The influence of conflicting response mappings on switch costs is well established in the task-switching literature. Responses are typically faster on congruent trials than on incongruent trials (Meiran, 1996; Rogers \& Monsell, 1995). This is taken as evidence that stimulus-response mappings for both tasks are activated on every trial, even though one is irrelevant. Furthermore, this congruence effect interacts with the switch cost, with larger switch costs on incongruent trials (Monsell et al., 2000). This suggests that switching task is harder when there is increased response conflict. Indeed, switch costs seem largely dependent on the conflict created by bivalent stimuli and responses as they are greatly reduced when there is no overlap between the two tasks (Kray \& Eppinger, 2006; Mayr, 2001; Rogers \& Monsell, 1995).

Previous research comparing children to adults has suggested that children are particularly susceptible to interference effects in task-switching. Cepeda et al. (2001) found that while adults benefited from increasing the time between the response and the subsequent trial (controlling for advance preparation time), children did not. This suggests that children experience more persisting interference from the S-R mappings that were relevant on the previous trial. Further evidence that children experience greater interference from irrelevant stimulus-response mappings came from their finding that children (aged 10 years and above) 
experience greater congruence effects than adults $^{2}$. A study by Crone, Bunge, van der Molen and Ridderinkhof (2006) found that children show a greater reversed repetition effect than adults, i.e. they find it harder to switch to a new task when repeating the same response, compared to switching both task and response. Crone et al. suggested this is because children experience greater carry-over effects from the previously activated S-R associations.

This research has suggested that children are more susceptible to interference effects in task-switching than adults, implying that the ability to control interference from taskirrelevant information improves with age. Yet the development of this ability within childhood has not been investigated to confirm this. We addressed this issue in Experiment 1, examining how the effects of interference from task-irrelevant information in the taskswitching paradigm change with age during mid-childhood. Experiment 2 explored this further, investigating what aspects of interference might account for developmental change.

\section{Experiment 1}

This experiment compared two groups of children, 5- to 8-year-olds and 9- to 11-yearolds, to investigate developmental changes in the ability to shift task-set as well as to determine how the ability to resist interference in the task-switching paradigm improves during childhood. One problem with many of the previous studies that have investigated task-switching in children is that the tasks were not designed specifically for use with a young population. The children may have found the stimuli unappealing and had trouble with the written cues, meaning they were not performing at their optimum level. Given the large number of trials and speeded responses needed to gain a reliable and accurate measure of RT for each condition, it is important that the children are sufficiently motivated to maintain optimum performance throughout the task. To help the children engage with the task, we used a developmentally-appropriate

\footnotetext{
2 The term compatibility rather than congruence was used by Cepeda et al. (2001) however the principle is the same.
}

task-switching paradigm which involved making decisions about either the colour or pattern of a footballer's shirt. The colour and pattern tasks used the same stimuli and responses, creating both congruent and incongruent trials. We predicted that the influence of incongruent stimulus-response mappings would reduce with age, reflecting an increasing ability to resolve competition between the tasks. The task was performed across two sessions to reduce the demands placed on the children. This meant that we could also examine the effects of practice on children's task-switching performance.

\section{Method}

\section{Participants}

Ninety children participated in this experiment, divided into two age groups of 45 children each: 5- to 8-year-olds ( $M=6.98$, $S D=0.93 ; 22$ male) and 9- to 11-year-olds $(M=10.1, S D=0.80 ; 23$ male). The children attended state primary schools in middle-class, predominantly Caucasian neighbourhoods. All children had English as a first language. Bilingual children and those with a statement of special educational needs were excluded from the study. Informed parental consent was received for all children that participated.

\section{Materials and Procedure}

The experimental tasks were created and controlled using E-Prime software and run on a Dell laptop computer. This was connected to an ELO touchscreen placed approximately $270 \mathrm{~mm}$ from the edge of the table. An ergonomic mouse mat centred in front of that the touchscreen acted as a hand-rest. The children were asked to begin by placing their dominant hand on the hand-rest. This hand was used to respond on all trials. The taskswitching paradigm was split equally between two sessions, at least a day apart, and the blocks were interspersed with other tasks (not reported here) to keep motivation levels high. The order in which the sessions were completed was counterbalanced across participants.

The paradigm was introduced as a football match scenario in which the child had to choose which team a player was on. The target stimuli were footballers who were identical apart from the shirts that they were 
wearing. These were either spotty or stripy and could either be pink or yellow. The two tasks entailed making a decision based on either the colour or the pattern of the shirt.

On each trial, participants first saw the background scene of the 'football stadium' (Figure 1) After $1000 \mathrm{~ms}$ a cue appeared, indicating which task should be performed on that trial. For the colour task the cue consisted of a pink rectangle on the left of the screen and a yellow rectangle on the right, while for the pattern task this was a spotty rectangle on the left and a stripy rectangle on the right. $500 \mathrm{~ms}$ after the cue, the footballer appeared in the centre of the screen. Both the cues and the target remained on the screen until a response was made by touching one of the cue rectangles. The computer recorded the accuracy and latency of the response.

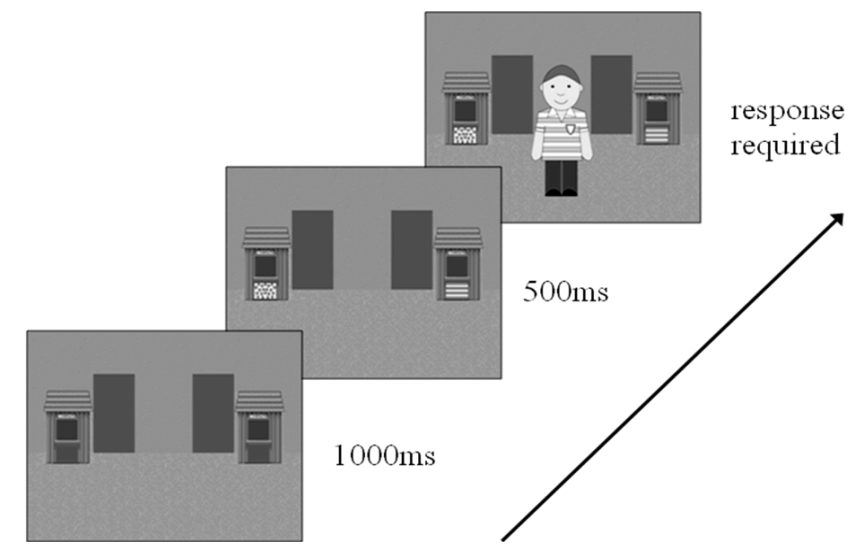

Figure 1:.Example of a pattern trial from Experiment 1. The stripes on the footballer's shirt would either be pink or yellow.

In each session the children first completed two pure blocks, one of colour trials and one of pattern trials, counterbalanced across participants and sessions. It was explained that they had to send the player through the correct door by touching the cue rectangle that matched his shirt. They were asked to do this as fast as possible and then return their hand to the hand-rest. Eight practice trials were performed followed by 64 test trials. Half-way through and at the end of each test block a feedback screen told the child how many correct responses they had made.

Following the pure blocks, the children performed mixed blocks in which the task (colour or pattern) and the target were selected randomly with equal probabilities so that $50 \%$ of trials were switch trials. The children completed two mixed blocks of 64 trials in each session (four in total). Pure trials were defined as trials from pure blocks in which just one task was performed. Switch trials were defined as trials in mixed blocks on which the task differed from the previous trial. Nonswitch trials occurred when the task repeated within the mixed block. The congruence of a trial was defined relative to the location in which the cues appeared. Pink cues and spotty cues were always on the left, and yellow and stripy cues were always on the right. This meant that trials on which the target was pink spots or yellow stripes were classed as congruent trials, due to the fact that the response was the same regardless of the dimension attended to. Trials with pink stripy or yellow spotty targets were classed as incongruent trials as the two dimensions (colour and pattern) indicated different responses.

\section{Results}

The first two trials of each block of the task-switching paradigm were treated as 'warm-up' trials and were not analysed. In addition, any trial on which more than one touchscreen response was recorded $(2.12 \%$ of trials) was treated as an equipment failure and not included in the data analysis. The median RT was calculated for each participant in each condition, measured from the time the target stimulus appeared until a response was made. Overall error rates were low, amounting to only $0.98 \%$ across age groups and conditions. Error trials were excluded from the RT analysis.

Two mixed-measures ANOVAs with trialtype (pure, non-switch, switch) as a within-subject factor and either gender (male, female) or order (colour first, shape first) as a between-subjects factor showed that there was no effect of gender or the order in which the blocks were completed $(F(1,88)<1, n s$, for both gender and order). Therefore these were not included as factors in further analyses.

\section{Mixing Costs}

General mixing costs were computed for each participant by subtracting the RT of all trials in pure blocks from non-switch trials in mixed blocks. To control for differences in baseline speed between participants, proportion mixing costs (non-switch RT / pure 
RT) were also calculated. Three-way, mixedmeasures ANOVA were performed for both the raw and proportion mixing costs. Task (colour, pattern) and Session (one, two) were included as within-subject factors and Age (5to 8-year-olds, 9- to 11-year-olds) was included as a between-subjects factor. As shown in Figure 2, the 9- to 11-year-olds showed smaller mixing costs than the 5- to 8year-olds as demonstrated by a significant main effect of Age (raw mixing cost: $F(1$, $88)=18.8, p<.001, \eta^{2}=.176$; proportion mixing cost: $\left.F(1,88)=11.7, p=.001, \eta^{2}=.118\right)$. A main effect of Task (raw mixing cost: $F(1,88)=7.63$, $p<.01, \eta^{2}=.080$; proportion mixing cost: $F(1$, $\left.88)=14.2, \quad p<.001, \quad \eta^{2}=.139\right)$ showed that mixing costs were larger for the colour task than for the pattern task (Figure 2). Mixing costs were smaller in session two for the 5- to 8-year-olds (raw: session 1: $M=186, S D=204$, $C I=136-208 ;$ session 2: $M=112, \quad S D=115$, $C I=95.2-155$; proportion: session 1: $M=1.17$, $S D=.17, C I=1.13-1.21$; session 2: $M=1.11$, $S D=.11, C I=1.08-1.14)$ but not the 9- to 11year-olds (raw: session 1: $M=61.3, S D=69.1$, $C I=16.2-106$; session 2: $M=61.1, S D=72.4$, $C I=32.6-89.6$; proportion: session $1: M=1.07$, $S D=.08, C I=1.03-1.11$; session 2 : $M=1.08$, $S D=.09, \quad C I=1.05-1.11)$ as shown by a significant interaction between Session and Age (raw mixing cost: $F(1,88)=4.39, p<.05$, $\eta^{2}=.047$; proportion mixing cost: $F(1$, 88) $\left.=3.94, p=.05, \eta^{2}=.043\right)$.
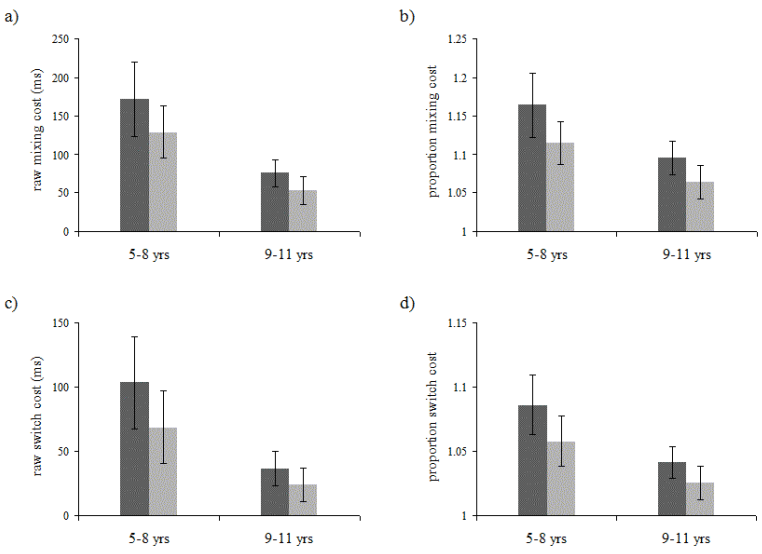

Figure 2: Figure 2. Mean a) raw and b) proportion mixing costs and c) raw and b) proportion switch costs for colour and pattern tasks in Experiment 1. Error bars represent $95 \%$ confidence intervals.

\section{Switch Costs}

Specific switch costs were computed by subtracting the RT on non-switch trials from the RT on switch trials. To control for differences in baseline speed between participants, proportion switch costs (switch RT / non-switch RT) were also calculated. Three-way, mixed-measures ANOVA were carried out for both the raw and proportion switch costs. Task (colour, pattern) and Session (one, two) were included as withinsubject factors and Age (5- to 8-year-olds, 9to 11-year-olds) was included as a betweensubjects factor. As shown in Figure 2, switch costs decreased as the children got older, as shown by a main effect of Age (raw switch cost: $\quad F(1, \quad 88)=14.7, \quad p<.001, \quad \eta^{2}=.143$; proportion switch cost: $F(1,88)=13.8, p<.001$, $\left.\eta^{2}=.136\right)$. As shown in Figure 2, switch costs were larger for the colour task compared to the pattern task (raw switch cost: $F(1,88)=5.37$, $p<.05, \eta^{2}=.058$; proportion switch cost: $F(1$, $\left.88)=7.51, p<.01, \eta^{2}=.079\right)$. There was no difference in switch cost across the two sessions, (raw mixing cost: $F(1,88)<1$,ns; proportion mixing cost: $F(1,88)<1$, ns).

\section{Congruence}

Initial analyses found that there was no effect of task on congruence and so a four-way mixed-measures ANOVA was performed with Task (colour, shape), Trialtype (pure, nonswitch, switch), Congruence (congruent, incongruent) and Session (session one, session two) as within-subject factors and Age (5- to 8-year-olds, 9- to 11-year-olds) as a betweensubjects factor. The Greenhouse-Geisser correction was used when the sphericity assumption was violated.

Responses were slower on incongruent trials $(M=1070, S D=281, C I=1024-1116)$ than congruent trials $(M=1034, S D=261, C I=991$ 1077 ) as shown by a significant main effect of Congruence, $F(1,88)=48.0, p<.001, \eta^{2}=.353$. This shows that the children responded faster when the two dimensions of the target indicated the same response than when they indicated conflicting responses. As shown in Figure 3, this was qualified by a significant Congruence $\mathrm{x}$ Age interaction, $F(1,88)=9.89$, $p<.01, \eta^{2}=.101$. This occurred because of larger congruence effects in the 5- to 8-yearolds than in the 9- to 11-year-olds ${ }^{3}$. A

3 To control for differences in baseline speed between the two age groups, the congruence analyses were repeated using logarithm-transformed RTs. Using these transformed RTs, the mean differences were 
significant Congruence $\mathrm{x}$ Trialtype interaction, $F(1.67,147)=15.7, p<.001, \eta^{2}=.151$, showed that the influence of congruence had a greater effect on switch trials than on non-switch trials. Interference from response mappings had less of an effect on performance in session two as shown by a significant Congruence $\mathrm{x}$ Session interaction, $F(1,88)=18.9, \quad p<.001$, $\eta^{2}=.177$.

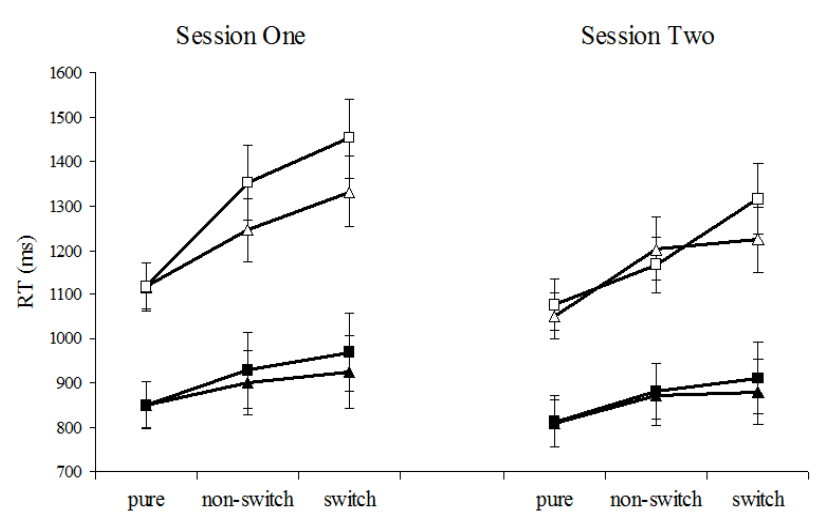

$\neg-5$-8yrs congruent $\longrightarrow \square-5$-8yrs incongruent $\rightarrow-9-11 \mathrm{yrs}$ congruent $\rightarrow-9-11 \mathrm{yrs}$ incongruent

Figure 3. Congruence effects for each trialtype in Experiment 1. Error bars represent 95\% confidence intervals.

Significant three-way interactions between Session, Trialtype and Congruence $(F(1.50$, $\left.132)=10.1, \quad p<.001, \quad \eta^{2}=.103\right), \quad$ Session, Congruence and Age $(F(1,88)=8.06, p<.01$, $\left.\eta^{2}=.084\right)$, and Trialtype, Congruence and Age $\left(F(1.67,147)=3.51, \quad p=.05, \quad \eta^{2}=.038\right) \quad$ were qualified by a significant four-way interaction between Age, Trialtype, Congruence and Session, $F(1.50,132)=5.91, p<.01, \eta^{2}=.063$. Tests of simple effects showed that while the 9- to 11-year-olds only showed a congruence effect on switch trials, the 5- to 8-year-olds showed a congruence effect on non-switch trials in session one, and on pure trials in session two ${ }^{4}$ (Figure 3).

The congruence results show that children's responses are influenced by the

equivalent to ratio scores and therefore less sensitive to baseline differences in performance (Kray, Li, \& Lindenberger, 2002; Kray \& Lindenberger, 2000; Meiran, 1996). The $\log$ RT analyses showed a very similar pattern of results to the median analyses, suggesting that any differences between the age groups were not due to differences in overall speed.

${ }^{4}$ The mean $\log$ RT results suggested that the 9- to 11-year-olds may also be influenced by congruence effects on pure trials in the second session. conflicting stimulus-response mappings of the task, supporting the findings of Cepeda et al. (2001). The congruence effect interacted with the switch costs, such that responses were slowest on incongruent trials that also required a switch in the task being performed. Overall, the younger children were more strongly influenced by interference from the irrelevant task, indicating that the ability to inhibit irrelevant information develops during midchildhood. Most interestingly, the congruence effect on pure trials in the second session suggests that once the children had become familiar with both tasks, the unattended dimension interfered with performance even when it was not required within the block. This demonstrates the pervasive influence that overlapping responses have on children's performance in the task-switching paradigm.

\section{Discussion}

This experiment used a developmentallyappropriate task-switching paradigm to investigate improvements in shifting task-set during childhood and explore how interference between tasks affects children's performance. The results showed a developmental improvement in task-switching within midchildhood, with 5- to 8-years-olds showing significantly larger mixing and switch costs than 9- to 11-years-olds. This demonstrates that the ability to maintain two task-sets in working memory, and also the ability to flexibly shift between them, develops during this period. Some researchers have argued that a global improvement in processing speed is responsible for all improvements during development (Hale, 1990; Kail, 1986, 1991). However, the difference in switch costs between the two age groups in this experiment remained significant even when differences in baseline speed were taken into account, suggesting that a general improvement in processing speed was not driving the agerelated changes in shifting task-set. Our results are consistent with the growing body of literature documenting the improvement of setshifting abilities in childhood (Chelune \& Baer, 1986; Cinan, 2006; Cohen et al., 2001; Crone et al., 2004; Dibbets \& Jolles, 2006; Huizinga et al., 2006; Schouten et al., 2000; Welsh et al., 1991).

The mixing and switch costs in Experiment 1 were asymmetric, due to a larger cost in RT 
on the easier colour task. This is consistent with the findings of Ellefson et al. (2006) who showed a similar pattern of switch costs on a colour-shape task-switching paradigm in both children and adults. Asymmetric switch costs have been attributed to greater persisting interference from the stronger task than the weaker task, such that extra inhibition of the stronger task is needed in order to perform the weaker task. This inhibition then has to be overcome when the stronger task is switched back to, resulting in a longer reaction time (Allport et al., 1994). The switch costs can also appear smaller in the stronger task, not because of a difference in RT on switch trials, but simply because participants are faster on nonswitch trials in this easier task. Mixing costs, but not switch costs, were smaller in session two for the 5- to 8-year-olds only. This suggests that practice improves the ability to maintain two task-sets in working memory, but not the ability to flexibly shift between them and has a greater effect in younger children.

We explored the influence of conflicting stimulus-response mappings on task-switching performance by examining the congruence effects; comparing trials where the tasks require the same, or different responses. Cepeda et al. (2001) found that children (aged ten years and above) showed a larger congruence effect than adults, suggesting that they find it harder to resolve the conflict between the two tasks. We added to this result by showing that the ability to overcome interference from irrelevant stimulus-response mappings improves during mid-childhood, as shown by smaller congruence effects in 9- to 11-year-olds compared to 5- to 8-year-olds. The ability to overcome this interference depended on the type of trial. Overall, the children found this more difficult on trials in which the task switched, presumably because the information they needed to inhibit had been relevant on the previous trial. In addition, the younger children were more susceptible to interference on incongruent trials where a task switch was not involved. This included trials in pure blocks in the second session during which the irrelevant task was never performed. This suggests that there was interference from the irrelevant task even when it was not being actively maintained as a possible response mapping for trials in that block, implying that the younger children were having greater difficulty inhibiting irrelevant information.

In summary, Experiment 1 replicated previous findings and showed that the ability to flexibly shift between tasks improves during mid-childhood. It also extended research comparing children and adults to show that the ability to resolve competition from conflicting task information improves during childhood. There are two possible levels at which conflict from the irrelevant task could be causing difficulty: At the perceptual level, children may find it difficult to focus their attention on only one dimension of the stimulus. Furthermore, conflict at the level of the responses may also interfere with children's performance. In Experiment 1 the tasks conflicted at both of these levels, making it difficult to determine the relative contribution of stimulus and response related conflict on children's shifting performance. In Experiment 2 we explored this question, orthogonally manipulating stimulus and response conflict to determine their relative contribution to children's performance.

\section{Experiment 2}

Currently, very little is known about which aspects of the overlapping tasks produce interference effects in school-age children. Findings from adults have suggested that conflict at the level of the stimulus and the response both influence the ability to shift task-set. In contrast, only stimulus conflict appears to affect switching performance in younger preschool children. This suggests that the extent to which stimulus and response conflict affect switching performance may change with age. In this experiment, we explored these developmental changes by examining how stimulus and response conflict affect school-age children's ability to switch between tasks.

\section{Stimulus and response conflict in adults}

Research in adults has suggested that stimulus and response conflict have independent effects on switching task. Meiran (2000) put forward a model whereby representations of stimuli and responses contribute to independent task-sets; a stimulus task-set controls the representation of the target stimuli, emphasising the relevant dimension compared to the irrelevant 
dimension, and a response task-set controls the representation of available responses. Response selection is then achieved via the interaction of stimulus and response codes. Meiran's model has received some support in the literature (Brass et al., 2003; Crone et al., 2006; Koch \& Allport, 2006; Sohn \& Anderson, 2003) although evidence from fMRI has suggested that response recoding may not be independent from task preparation (Brass et al., 2003).

Further evidence that conflict at the level of the stimulus affects the time taken to switch to a new task comes from Allport and colleagues (Allport \& Wylie, 2000; Koch \& Allport, 2006; Waszak et al., 2003) who showed that stimulus-based priming of a competing task contributes to the switching cost. In contrast to these findings, Rubin and Meiran (2005) did not find an effect of stimulus conflict on switch costs when they compared univalent and bivalent target stimuli. They suggest the discrepancy in findings may be because Waszak et al. (2003) and Allport \& Wylie (2000) used the alternating runs paradigm, however this explanation does not extend to Koch and Allport's (2006) results. An alternative explanation is that Rubin and Meiran used univalent responses in contrast to the typical bivalent responses used in the other studies. This suggests that there may be something important about concurrent conflict at both a stimulus and response level.

While they did not find any effects on switch costs, Rubin and Meiran (2005) did show an effect of stimulus conflict on mixing or global costs, the difference between RT in blocks of pure trials and blocks where both tasks were performed. From these results they argued that activation from the competing task in bivalent stimuli interferes with a task decision process that takes place on every trial in mixed blocks, including non-switch trials. This is consistent with Liston, Matalon, Hare, Davidson, and Casey (2006) who found that increased stimulus conflict slowed both switch and repeat trials, whereas increasing response conflict only affected switch trials. Experiments by Mayr and colleagues (Mayr, 2001; Mayr \& Bryck, 2007) have also suggested that reducing stimulus and response conflict has a greater effect on global costs than on switch costs. However, Mayr defined global selection costs as the difference between blocks of univalent and bivalent stimuli with two tasks performed in both conditions, rather than comparing blocks in which a single task is performed to blocks in which two tasks are performed, which is how global or mixing costs are usually calculated.

In summary, the adult task-switching literature provides mixed results as to the influence of stimulus and response conflict on switching task. Given that manipulations in parameters such as the stimuli used and the timing of events within a trial are known to influence the time taken to switch task, and these often vary between studies, it is not surprising that there are variations in findings. Nevertheless, the literature does show that under certain conditions interference at the level of the stimulus, and at the level of the response, contribute to the cost involved in switching to a different task.

\section{Stimulus and response conflict in children}

The effects of stimulus and response conflict have been investigated in preschoolers using the Dimensional Change Card Sort test (DCCS). This is similar to the task-switching paradigm and involves sorting cards according to either the colour or shape of the picture on the card. There is only one switch involved in the DCCS, and while 4-year-olds have no problem sorting according to a new dimension, many 3-year-olds continue to sort using the previous rule (Kirkham, Cruess, \& Diamond, 2003; Perner \& Lang, 2002; Zelazo, Frye, \& Rapus, 1996). As the stimuli and responses for the two tasks overlap in a similar manner to the task-switching paradigm a number of studies have addressed the effect of stimulus and response conflict on preschoolers' ability to switch and sort the cards by a new rule.

Reducing conflict at the stimulus level has been shown to help performance on the DCCS. Perner and Lang (2002) showed that compared to the standard version, children had no problem switching in a 'same-silly' game using univalent cards displaying one of two objects of the same colour. They argued this was because the children no longer needed to re-describe the cards according to the new dimension. Brooks, Hanauer, Padowska and Rosman (2003) claimed that this manipulation also reduced the complexity of the stimuli, which may have made it easier to selectively attend to the relevant dimension. Brooks et al. found that if the target stimuli varied on the irrelevant colour dimension, accuracy on the 
'same-silly' game decreased. They argued that this is because children's difficulty lies in selectively attending to the relevant dimension rather than in re-describing the stimulus in a new way.

The findings of Brooks et al. (2003) have not always been replicated (Kloo, Perner, Kerschhuber, Dabernig \& Aichhorn, 2008). However, several studies have shown that children are greatly affected by variation on an irrelevant dimension of a stimulus when making speeded classifications on another dimension, known as the Garner interference effect (e.g. Garner, 1970; Garner \& Felfoldy, 1970). Moreover, the interference from the irrelevant dimension reduces with age (Ridderinkhof, van der Molen, Band, \& Bashore, 1997; Shepp \& Barrett, 1991; Strutt, Anderson, \& Well, 1975), suggesting a developmental improvement in overcoming interference from conflicting information.

Interference from the irrelevant dimension is generally found to decrease if it is a property of a separate object, rather than a feature of the same stimulus as the relevant dimension (Garner \& Felfoldy, 1970; Shepp \& Barrett, 1991). This idea was used by Kloo and Perner (2005) to test their theory that children have difficulty re-describing stimuli in the DCCS. They used cards where an outline of an object was presented next to a coloured circle so that the two dimensions were no longer properties of the same object. Performance improved when the dimensions on the test cards (cards to be sorted) were separated, but not when the target cards were separated (but see Zelazo, Müller, Frye, \& Marcovitch, 2003; Experiment 6).

The attentional inertia theory (Diamond \& Kirkham, 2005; Kirkham et al., 2003; Kirkham \& Diamond, 2003) is similar to the redescription theory (Kloo \& Perner, 2005; Perner \& Lang, 2002) in that it proposes that once children have thought of a stimulus in terms of one dimension, e.g. its shape, they have great difficulty in disengaging from this dimension. Diamond, Carlson and Beck (2005) proposed that if the two dimensions were not properties of the same object then this difficulty would be reduced. They included a condition whereby a black object was presented on a card with a coloured background. This was a more stringent test than that used by Kloo and Perner (2005), as there was less separation between the dimensions, meaning that the use of spatial attention was not as easily available as a strategy to select the relevant dimension. In addition, colour was a property of the background, surrounding the object on all sides, rather than a feature of a separate object. The dimensions were separated on both test and target cards. While there was no difference for $2^{1 / 2}$-year-olds, the performance

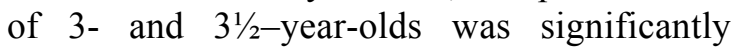
improved when the dimensions were separated. This supports the findings of Kloo and Perner (2005) suggesting that preschoolers find switching task easier when the same object does not have to be seen in two ways i.e. when stimulus conflict is reduced.

It should be noted that in these experiments, even when the stimuli on the target cards were separated, the same two response locations were used for both tasks. Towse, Redbond, Houston-Price and Cook (2000) included a condition in the DCCS whereby four response locations were used so that responses for the two tasks did not overlap. Although this did not improve performance, this finding is difficult to interpret as the majority of children (91\%) passed the standard version, meaning that there was not much room for improvement. In a sample of a similar age but with only a $10 \%$ pass rate on the standard condition, Rennie, Bull and Diamond (2004) also found that using univalent responses did not significantly improve performance. Taken together, these studies show that reducing response conflict does not help preschoolers to switch task on the DCCS.

There is a discrepancy between the findings from adults and preschoolers such that while both age groups benefit from reduced conflict at a stimulus level, only the adults benefit from reduced conflict at the level of the response. Little is known about the influence of stimulus and response conflict on school age children's ability to flexibly switch between tasks. Are they only helped to switch by reducing conflict at a stimulus level, as for preschoolers? Or, like adults, does reducing response conflict also help switching performance?

In Experiment 2 we orthogonally manipulated stimulus and response conflict by varying the overlap of stimulus dimensions and response mappings in a new version of the task-switching paradigm. New stimuli were 
used, as it was not possible to separate the stimulus dimensions on the footballer stimuli used in Experiment 1. The effects on both switching costs and congruency effects were examined. Based on previous research (e.g. Meiran, 2000) our initial predictions were that stimulus and response conflict would have independent effects, so that separating either stimulus or response dimensions would reduce switch costs. Furthermore we predicted that these would have an additive effect so that performance would be most improved when there was no conflict between tasks at either level. We also predicted that reducing response conflict would reduce the congruency effect as this is largely caused by overlapping response mappings. To determine if the effects of stimulus and response conflict change with age we compared two groups of children, 5- to 7-year-olds and 9- to 11-yearolds. We expected that switch costs and congruency effects would be smaller in the older age group and that greater agedifferences would be seen at high levels of stimulus and response conflict.

\section{Method}

\section{Participants}

A new sample of 60 children took part in Experiment 2, thirty 5- to 7-year-olds $(M=6.37, S D=0.58 ; 14$ male $)$ and thirty 9 - to 11 -year-olds $(M=10.35, S D=0.60 ; 15$ male). The children attended state primary schools in middle-class, predominantly Caucasian neighbourhoods. All children had English as a first language. Bilingual children and those with a statement of special educational needs were excluded from the study. Informed parental consent was received for all children that participated.

\section{Materials and Procedure}

The experimental set-up for Experiment 2 was the same as Experiment 1. The target stimulus was a picture of a cupcake presented on a dark red screen along with a number of shopping baskets that served as the response locations. The cakes varied on two dimensions: colour (purple or green), and the shape on top of the cake (flower or star). As shown in Figure 4, the level of overlap for the two stimulus dimensions was manipulated so that either the shape itself was coloured and the top of the cake was white (integrated stimulus: SI) or the top of the cake was coloured and the shape on top was white (separated stimulus: SS). This was similar to the stimulus separation used by Diamond et al. (2005) where the shape is a property of the foreground and the colour a property of the background.

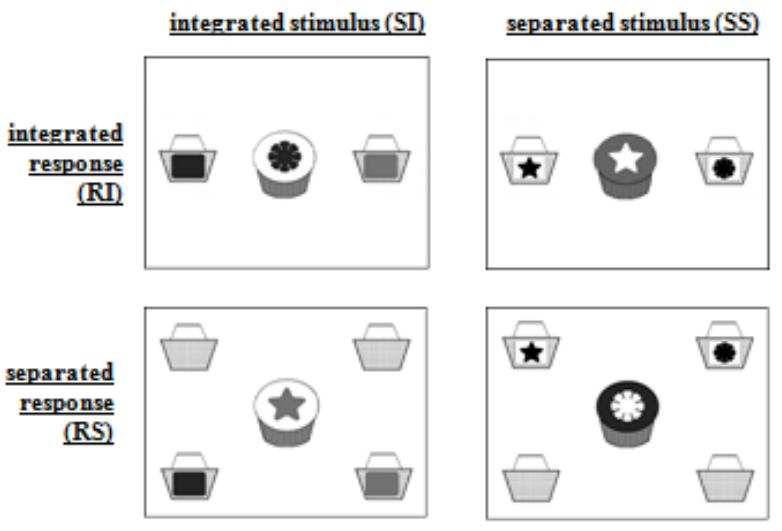

Figure 4. Example of the four conditions used in Experiment 2. For the colour cues and top of the cakes, black represents purple and grey represents green. For the real stimuli the background was dark red.

The cake was matched to a basket on the basis of either its colour or its shape according to the cue presented at the start of the trial. The cues consisted of rectangles superimposed on the shopping baskets at the possible response locations. Response overlap for the two tasks was also manipulated. In the integrated response (RI) condition there were only two shopping baskets, presented centrally either side of the cake. In the separated response (RS) condition four shopping baskets were presented, one in each corner of the screen. If the cake was to be matched on colour then in the RI condition a purple rectangle was displayed on the left basket and a green rectangle on the right. In the RS condition the colour cues were displayed on the bottom two baskets. If the cake was to be matched on its shape then a solid black star was displayed on a white rectangle on the left basket, and a solid black flower was displayed on a white rectangle on the right basket. In the RS condition these were presented on the top two baskets. The four conditions were combined to create four blocks of trials: separated stimulus, separated response (SS, RS), separated stimulus, integrated response (SS, RI), integrated stimulus, separated 
response (SI, RS), and integrated stimulus, integrated response (SI, RI).

The experiment was split across two sessions at least one day apart. In Session A the SI, RS block was administered first followed by the SI, RI block. The two blocks were separated by approximately ten minutes during which time other tasks (not reported here) were administered. In session B the SS, RI block was followed ten minutes later by the SS, RS block. The order in which the two sessions were completed was counterbalanced across participants so that half the children completed Session A followed by Session B (order one), and the other half completed Session B first (order two). There were no pure blocks of trials (cf. Experiment 1).

During the testing phase the baskets were always present on the screen. At the start of a trial the cues appeared for $500 \mathrm{~ms}$, followed by the target stimulus. The task (colour, shape) and the target were selected randomly with equal probability on each trial. The cues remained on the screen after the target appeared and a response was then made by touching the cue that matched the cake. In the RS condition it was emphasised to the child that they must touch a basket that had a picture on it and not an empty basket. The interval between the response and the start of the next trial was $1000 \mathrm{~ms}$ and the children were asked to return their hand to the hand-rest during this time. The children completed eight practice trials followed by 112 test trials in each block. Halfway through and at the end of each block a feedback screen displayed how many correct responses the child had made.

\section{Results}

Switch and non-switch trials were defined as in Experiment 1. The trials were also coded for congruence. Even when response locations were separated, stars and purple stimuli always required a response on the left hand side of the screen, while flowers and green stimuli always required a response on the right hand side of the screen. A congruent trial was defined as a trial where both dimensions of the target required a response on the same side of the screen. An incongruent trial was defined as a trial where the two dimensions indicated responses on opposite sides of the screen. The data were treated as in Experiment 1. Errors were rare, amounting to $0.96 \%$ of trials and only $0.75 \%$ of trials were excluded due to equipment error. A mixed-measures ANOVA with trialtype (non-switch, switch) and gender (male, female) as factors showed that there was no effect of gender, $F(1,58)<1$, ns, therefore this factor was not included in further analyses.

To ensure that switch costs and congruence effects were present, a four-way mixedmeasures ANOVA with Age (5- to 7-yearolds, 9- to 11-year-olds) as a between-subjects factor and Trialtype (switch, non-switch), Congruence (congruent, incongruent) and Task (colour, shape) as within-subject factors was performed. A significant main effect of Trialtype, $F(1,58)=53.8, \quad p<.001, \eta^{2}=.481$, and also an interaction between Trialtype and Age, $\quad F(1, \quad 58)=10.2, \quad p<.01, \quad \eta^{2}=.150$, confirmed that switch costs decreased with age, replicating Experiment 1. The children were faster on congruent compared to incongruent trials, $F(1,58)=13.6, \quad p<.001$, $\eta^{2}=.190$, however the two age groups did not differ, $F(1,58)<1, n s$. Switch costs were larger on incongruent trials, $F(1,58)=4.18, p<.05$, $\eta^{2}=.067$, as found in Experiment 1 .

A main effect of Task demonstrated that responses were faster for the colour than the shape task, $F(1,58)=88.6, p<.001, \eta^{2}=.604$, particularly for the younger age group, $F(1$, $58)=8.80, p<.01, \eta^{2}=.132$, suggesting that the children found the colour task easier. A significant interaction between Trialtype and Task, $F(1,58)=6.84, p<.05, \eta^{2}=.106$, identified a larger switch cost in the colour task $(50 \mathrm{~ms})$ than the shape task $(24 \mathrm{~ms})$. This is consistent with the results in Experiment 1 and Ellefson et al. (2006) in showing asymmetric switch costs between colour and shape tasks. Furthermore, significant congruence effects were present in the colour task $(29 \mathrm{~ms})$ but not the shape task $(3 \mathrm{~ms}), F(1,58)=5.88, p<.05$, $\eta^{2}=.092$.

As task had an important influence on performance, we decided to investigate the influence of stimulus and response overlap on switch costs and congruence effects for each task separately. For switch costs, two threeway mixed-measures ANOVAs with Age (5to 7-year-olds, 9- to 11-year-olds), Trialtype (switch, non-switch) and either Response Overlap (RI, RS) or Stimulus Overlap (SI, SS) were run for each task (Colour and Shape) separately. Similar ANOVAs were run to 
investigate congruence effects replacing Trialtype with Congruence (congruent, incongruent) as a factor. ${ }^{5}$

\section{The effects of stimulus and response conflict on switch costs}

For the shape task only, the influence of stimulus and response overlap on switch costs differed for the two age groups. While reducing response overlap lowered switch costs in the older children, $F(1,58)=4.54$, $p<.05, \eta^{2}=.073$, reducing stimulus overlap on the shape task lowered switch costs in the younger children, $F(1,58)=5.02, \quad p<.05$, $\eta^{2}=.080^{6}$. This could suggest that the older children were more influenced by response overlap on the shape task whereas the younger children were more influenced by stimulus overlap. It is worth noting that the reduction in switch costs in these cases is at least partly influenced by an increase in RT on non-switch trials, rather than a decrease in RT on switch trials (Figure 5).

a)
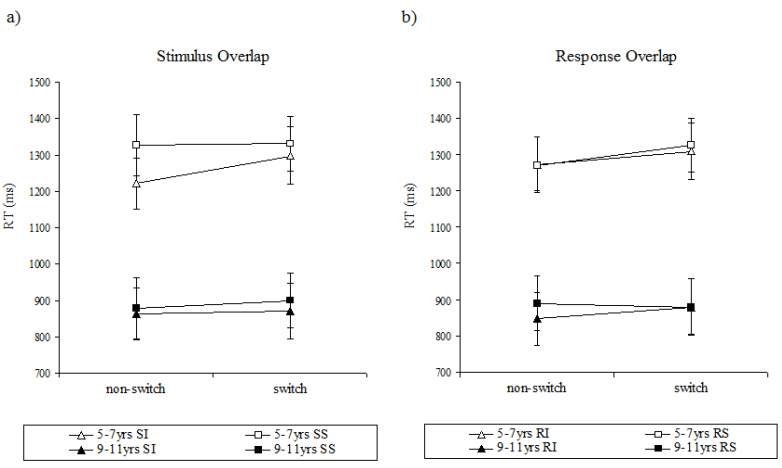

Figure 5. Mean RT for switch and non-switch trials in the shape task of Experiment 2 as a function of a) stimulus and b) response overlap. SI = integrated stimuli, $\mathrm{SS}=$ separated stimuli, $\mathrm{RI}=$ integrated responses, $\mathrm{RS}$ $=$ separated responses. Error bars represent $95 \%$ confidence intervals.

Order effects. Repeating the same analyses including Order (one, two) as a factor showed that the order in which the conditions were

\footnotetext{
${ }^{5}$ All analyses were repeated using logarithmtransformed RTs to control for differences in baseline speed between participants. These analyses showed a largely similar pattern of results to the median analyses, suggesting that any differences between the age groups were not due to differences in overall speed.

${ }^{6}$ The Age $x$ Trialtype $x$ Stimulus Overlap interaction did not reach significance when $\log$ RT was used as the dependent variable, $\mathrm{F}(1,58)=1.86$, $n s$.
}

performed mediated the effects of stimulus and response overlap in both tasks (Figure 6). Generally, the children were slower in the stimulus or response overlap condition that they encountered first. For the colour task, all children were slower on the stimulus overlap condition they encountered first, $F(1$, 56) $=18.3, p<.001, \eta^{2}=.246$, but only in the first response overlap condition if it was the RI condition (order two), $F(1,56)=8.60, p<.01$, $\eta^{2}=.133$. For the shape task, all children were slower on the response overlap condition they encountered first, $F(1,56)=17.1, p<.001$, $\eta^{2}=.234$, and the first stimulus overlap condition if it was the SS condition, $F(1$, 56)=17.8, $\quad p<.001, \quad \eta^{2}=.242$. These results suggest that performance is slower in the stimulus and response condition encountered first, but that this is partly dependent on the task and condition that is being performed.

More importantly, including order as a factor showed that separating responses did reduce switch costs in the colour task, but only for those children who did the RI condition first (order two), as shown in Figure 6b, $F(1$, $56)=8.60, p<.01, \eta^{2}=.133$. This shows that practice effects interacted with the effect of response conflict on switch costs.
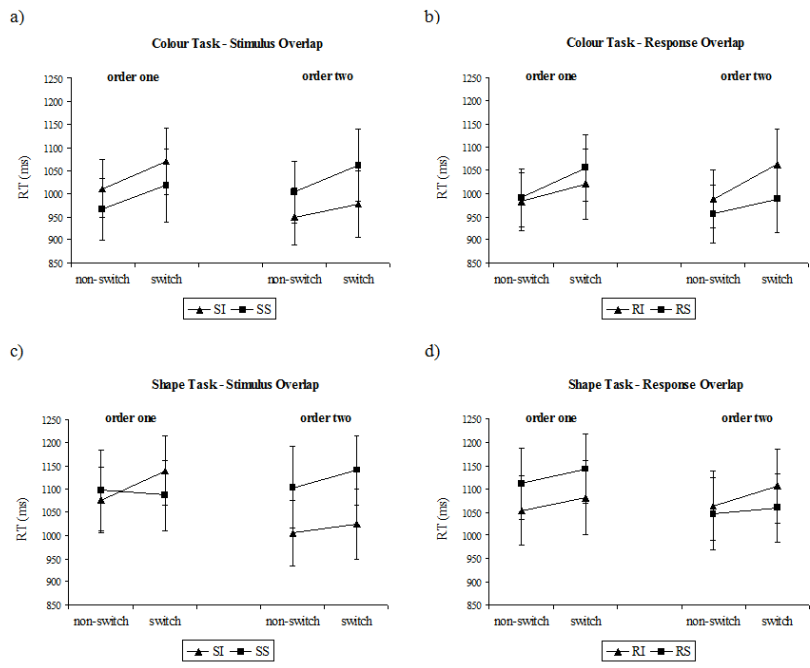

Figure 6. Order effects in Experiment 2 influence a) a) stimulus overlap and b) response overlap in the colour task and c) stimulus and d) response overlap in the shape task. SI = integrated stimuli, $\mathrm{SS}=$ separated stimuli, $\mathrm{RI}=$ integrated responses, RS =separated responses. Error bars represent $95 \%$ confidence intervals. 


\section{The effects of stimulus and response conflict} on congruence

The predicted interaction between Response Overlap and Congruence was present in the colour task (Figure 7b). Separating the responses reduced the congruence effect, $F(1,58)=6.83, \quad p<.05$, $\eta^{2}=.105$, however this did not interact with age, $F(1,58)=1.05$, $n s$.
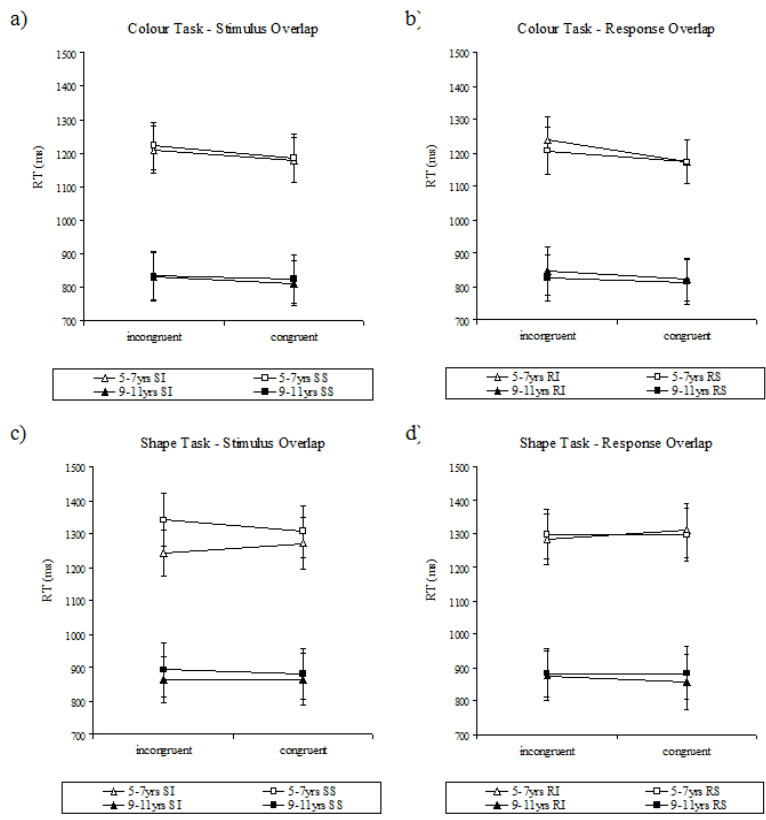

Figure 7. Congruence effects in Experiment 2 as a function of a) stimulus overlap and b) response overlap in the colour task and c) stimulus and d) response overlap in the shape task. SI = integrated stimuli, $\mathrm{SS}=$ separated stimuli, RI = integrated responses, RS =separated responses. Error bars represent $95 \%$ confidence intervals.

Despite there being no main effect of congruence on the shape task, $F(1,58)<1$, ns, there was a significant Stimulus Overlap $\mathrm{x}$ Congruence interaction, $F(1, \quad 58)=11.4$, $p=.001, \eta^{2}=.165$. This was due to the fact that responses were particularly slow on incongruent shape trials when the stimulus dimensions were separated (congruent: $M=1094, \quad S D=300, \quad C I=1039-1148$; incongruent, $M=1117, S D=312, \quad C I=1061$ 1173) but not when the stimulus dimensions were integrated (congruent: $M=1067, S D=293$, $C I=1013-1122 ; \quad$ incongruent, $\quad M=1053$, $S D=267, \quad C I=1004-1102$ ). $\quad$ Thus the congruence effect increased rather than decreased when colour was separated from shape. This shows that our manipulation of stimulus conflict did not work as we intended it to. A significant Stimulus Overlap $\mathrm{x}$ Congruence $\mathrm{x}$ Age interaction, $F(1,56)=5.43$, $p<.05, \eta^{2}=.086$, demonstrated that separating the stimuli increased interference from the colour task in the shape task more in the 5- to 7-year-olds than the 9- to 11-year-olds (Figure $7 \mathrm{c})$. The order in which the conditions were performed did not mediate the influence of stimulus and response conflict on congruence effects.

\section{Discussion}

This experiment set out to systematically examine the effects of stimulus and response conflict on children's ability to switch between tasks. We reduced response conflict by separating the response locations for the colour and shape tasks. At a stimulus level we attempted to reduce conflict by making shape a property of the foreground and colour a property of the background, following Diamond et al. (2005). Far from reducing stimulus conflict, in certain situations this manipulation actually seemed to increase conflict, particularly on the shape task. In addition to this unexpected effect, we were also surprised to find that both the task and the order in which stimulus and response conflict were manipulated had such an impact. The children found the colour task easier than the shape task, and the presence of asymmetric switch costs also suggested that colour was the stronger task. Consequently, there was much more interference from the colour task on the shape task than the other way round, particularly at the stimulus level. These results revealed the complex interplay of factors that affect children's ability to switch task. We discuss these factors for switch costs and congruence effects in turn.

\section{Switch costs}

Switch costs were smaller in the older children, replicating Experiment 1 and demonstrating that the ability to switch task improves during childhood. Our initial prediction was that stimulus and response conflict would have independent but additive effects on switch costs. However, the analyses revealed a more complicated pattern of results. Reducing stimulus and response overlap seemed to have independent effects on switch costs, but this depended on the relevant task and also the order in which the blocks were performed. Overall, separating task overlap at a response level reduced the switch cost in the 
older children on the shape task, and if the separated responses condition was done in the second block on the colour task. This suggests that it was most effective after practice with the tasks.

Developmental differences were seen in the shape task where the two age groups showed different patterns of switch costs: 9to 11-year-olds showed a reduction in switch costs when the responses were separated, whereas 5- to 7-year-olds showed equivalent switch costs both when the responses were separated and integrated. This suggests that they still experienced interference from the colour task even when the responses were separated. This finding is interesting given that separating response locations influences switch costs in adults (Brass et al., 2003; Meiran, 2000; but see Mayr, 2001) but not in preschoolers (Rennie et al., 2004; Towse et al., 2000). While this may due to the different paradigms used with different age groups, or because response conflict has a greater effect on RT than accuracy; however it could indicate that, as children get older, their ability to use univalent response mappings to separate task-sets at a conceptual level improves.

While separating responses on the shape task did not reduce switch costs in the younger children, separating the stimulus dimensions did. This seems to conflict with our observation that separating the stimuli increased the congruence effect on the shape task. It appears that separating the stimulus dimensions and putting colour as a property of the background made it more salient, increasing conflict between the tasks and creating interference on incongruent trials. The switch cost and congruence results can be reconciled by considering the fact that switch costs were only reduced when the stimulus dimensions were separated because non-switch trials were also slower. Therefore, this suggests that the younger children experienced interference from the colour task on all incongruent trials, whether a switch was required or not. This is consistent with the findings from Experiment 1, which demonstrated that the younger children showed a congruence effect on non-switch and pure trials, whereas the older children did not. It is relevant to note that Rubin and Meiran (2005) suggest that activation from the competing task at a stimulus level interferes with a task decision process that takes place on every trial, including non-switch trials. However Mayr (2001) proposed that only older adults need to select a relevant task-set on both switch and non-switch trials whereas young adults only do this on switch trials. A similar pattern seems to emerge in the shape task between younger and older children. While the younger children were slowed on both non-switch and switch trials when there was more task interference (in this case when the stimulus dimensions were separated), the older children only seemed to be slowed on switch trials, evidenced by the fact that their switch costs were not reduced.

\section{The effects of task order}

At a basic level, stimulus and response overlap affected overall speed. Responses were generally slower in those conditions that were encountered first, although this also depended on the task and the order in which the blocks were performed. More interestingly, order affected the influence that separating the responses had on switch costs in the colour task, such that reducing response conflict only helped if not encountered in the first block. The influence of order shows the importance of considering what are often assumed to be unimportant factors. Further work using a more sophisticated manipulation of order would determine if these effects are driven by practice or as a result of how the tasks are first encountered.

\section{Congruence}

Our prediction that separating the response locations would reduce the difference in RT between congruent and incongruent trials was supported for the colour task only, showing that interference from the response mappings for the shape task was reduced when the responses were separated. In the shape task, increased conflict from the colour task at the stimulus level may have counteracted the reduction in conflict caused by separating the response mappings.

Different patterns of development were also apparent for the colour and shape tasks. On the colour task, manipulating stimulus and response overlap produced largely the same effects for both age groups. However, on the shape task the younger children experienced more conflict from the irrelevant colour task. This was demonstrated by increased 
interference from colour when it surrounded the shape, and the fact that separating the response dimensions did not reduce interference from the irrelevant mappings and make it easier to switch tasks. This suggests that while all children were able to overcome the interference from the shape task when performing the easier colour task, the younger children struggled on the shape task when there was more irrelevant interference from the stronger colour task. Thus the ability to overcome interference from previous S-R mappings does improve during development. There was no developmental change in the congruence effect itself, in contrast to Experiment 1. Arguably, this may have been masked by the stimulus and response overlap manipulations.

\section{Why did separating colour from shape create more interference?}

Our results quite clearly showed a discrepancy in the interference between the two tasks. One explanation is that interference from the stronger colour task on the shape task was much larger than interference from the shape task on the colour task, and this mediated the influence of stimulus and response overlap on the two tasks. This is reminiscent of Ridderinkhoff, van der Molen, Band, and Bashore's (1997; Experiment 1) observations from an experiment using the Garner interference paradigm with 5- to 12 year-olds. They found larger interference and congruence effects on the colour task when the irrelevant dimension (orientation) was part of the same stimulus (vertical or tilted bar). However, consistent with our findings, interference from the colour task on the orientation task was greater when colour was a property of a square contour surrounding the bar. Together these findings show that two dimensions do not have to be part of the same object for conflict to occur. As colour is a particularly salient dimension which is likely to capture attention, separating it from the relevant dimension may draw attention away from the target, slowing processing of the relevant task even more than if colour was part of the same object. A further explanation is that it was more difficult to selectively attend to the relevant shape due to increased demands on space-based and object-based attentional mechanisms.
Given our findings, it is interesting that there are no task differences in the DCCS, where performance is equivalent for colour and shape, even when the stimuli are separated, as in our experiment. Furthermore, and in contrast to our results, Diamond, Carlson, and Beck (2005) found that switching performance improved when colour was a property of the background. There are a number of differences between our task and the DCCS that may explain the discrepancy in results. Firstly, in the DCCS only one task is performed per block, rather than continuous switching between tasks. It may be that the asymmetry in task interference only affects switch costs when it is necessary to keep both task-sets actively maintained. Secondly, when Diamond et al. separated colour and shape, the background of the sorting cards became coloured with a black shape superimposed on top. In our experiment colour was still a property of the cake, even though it was separated from the shape.

In addition, in Diamond et al.'s task, black shapes on the sorting cards had to be matched to black shapes on the target cards, whereas in our version, the shapes on the cakes were white and had to be matched to black shapes on the cues. Another possible explanation is that the target items in the standard DCCS also create a visual clash between the test and target items (e.g. when matching on shape there is a clash between the colours), which is not present in our experiment. Reducing this visual conflict by replacing the target items with puppets helps performance on the DCCS (Perner \& Lang, 2002). Using four target items also removes this visual conflict, but does not improve performance (Rennie et al., 2004; Towse et al., 2000). Therefore it seems unlikely that the visual clash between test and target items can explain the discrepancy between our results and the DCCS. There may be something about using puppets as targets that aids children's performance on the DCCS, in addition to removing the visual clash.

\section{Limitations and future directions}

As a result of task (shape vs. colour) differences, and the fact that by separating the stimulus dimensions we actually increased stimulus conflict rather than reduced it, addressing our initial hypotheses about the relative contribution of stimulus and response conflict is difficult. A better way to examine 
the effects of stimulus conflict may be to use univalent stimuli or to vary the perceptual similarity of the irrelevant feature compared to a prototype used as the target feature, for example by using a paler shade of the same colour (Liston et al., 2006).

In our experiment, reducing the overlap between the tasks at a stimulus and response level did affect switch costs. This is in contrast to some findings from the adult literature (Mayr, 2001; Mayr \& Bryck, 2007; Rubin \& Meiran, 2005), which suggest that manipulating stimulus conflict has a greater effect on mixing costs than on switch costs. Mixing costs could not be examined in this experiment as all blocks contained a mixture of trials. However, given that stimulus overlap also affected non-switch trials on the shape task in the younger children, this would be an interesting avenue to pursue. It would also be informative to manipulate the cue-target interval to investigate how the exogenous processes are affected by task preparation.

\section{General Discussion}

This study set out to investigate the factors affecting children's ability to shift between tasks and its development during midchildhood, in particular the role of task interference. Experiment 1 showed that as well as an improvement in the ability to flexibly shift between tasks, interference from the irrelevant task decreased with age. This suggests that as children get older they also get better at resolving conflict between interfering tasks. These skills are important in everyday situations, in order to flexibly attend to relevant information and ignore distractions from competing stimuli. During midchildhood, children are required to do exactly this in the classroom. Thus, the findings from Experiment 1 suggest that as children get older their general learning skills improve as they become more able to deal with many competing sources of information.

Experiment 2 extended the findings of Experiment 1 to look at the relative contribution of stimulus- and response-related interference on the ability to switch between tasks. This is important in determining whether children are more distracted by perceptual or response-related interference, and may have important implications for how best to reduce interference and distractions to help children learn. The results from Experiment 2 supported our previous finding that the ability to overcome interference from conflicting information improves during mid-childhood and affects children's ability to switch between tasks. Furthermore, it showed that both perceptual and response interference affects children's ability to flexibly shift between tasks. This experiment also suggested that the exogenous interference effects in the taskswitching paradigm are largely dependent on the specific tasks that are used. Interference from colour was particularly high, even when it was separated from the shape dimension. While further work is clearly required, this suggests that there may be particular situations or stimuli that children find particularly distracting.

In conclusion, this study demonstrates that both the ability to flexibly shift between different tasks, and overcome interference from an irrelevant task, improves during midchildhood. Furthermore, children's ability to flexibly shift attention is affected by interference at both the level of the stimulus, and the response, but also the specific tasks that are switched between. A better understanding of set-shifting development, and the factors that affect it, may have important implications for how to improve these skills, and create optimal situations in which children can learn most effectively.

\section{Acknowledgements/Author Notes}

This research was supported by a Biotechnology and Biological Sciences Research Council Studentship and Economic and Social Research Council Postdoctoral Fellowship Award PTA-026-27-1648. We thank the staff and pupils of the participating schools for their help and cooperation.

\section{References}

Allport, A., Styles, E. A., \& Hsieh, S. (1994). Shifting intentional set: Exploring the dynamic control of tasks. In C. Umilta \& M. Moscoviitch (Eds.), Attention and Performance $X V$ (pp. 421-452). Cambridge, MA: MIT Press.

Allport, A., \& Wylie, G. (2000). Task switching, stimulus-response bindings, and negative priming. In S. Monsell \& J. Driver (Eds.), Control of cognitive processes: Attention and Performance XVIII (pp. 35-70). Cambridge, MA: MIT Press. 
Brass, M., Ruge, H., Meiran, N., Rubin, O., Koch, I., Zysset, S., et al. (2003). When the same response has different meanings: recoding the response meaning in the lateral prefrontal cortex. NeuroImage, 20(2), 1026-1031.

Brooks, P. J., Hanauer, J. B., Padowska, B., \& Rosman, H. (2003). The role of selective attention in preschoolers' rule use in a novel dimensional card sort. Cognitive Development, 18(2), 195-215.

Cepeda, N. J., Kramer, A. F., \& Gonzalez de Sather, J. C. M. (2001). Changes in executive control across the life span: Examination of task-switching performance. Developmental Psychology, 37(5), 715-730

Chelune, G. J., \& Baer, R. A. (1986). Developmental norms for the Wisconsin Card Sorting Test. Journal of Clinical and Experimental Neuropsychology, 8, 219-228.

Cinan, S. (2006). Age-related changes in concept formation, rule switching, and perseverative behaviors: A study using WCST with 12 unidimensional target cards. Cognitive Development, 21(3), 377-382.

Crone, E. A., Bunge, S. A., van der Molen, M. W., \& Ridderinkhof, K. R. (2006). Switching between tasks and responses: a developmental study. Developmental Science, 9(3), 278-287.

Diamond, A. (2002). Normal development of prefrontal cortex from birth to young adulthood: Cognitive functions, anatomy, and biochemistry. In D. T. Stuss \& R. T. Knight (Eds.), Principles of frontal lobe function (pp. 466-503). Oxford: Oxford University Press

Diamond, A., Carlson, S. M., \& Beck, D. M. (2005). Preschool children's performance in task switching on the dimensional change card sort task: Separating the dimensions aids the ability to switch. Developmental Neuropsychology, 28(2), 689-729.

Diamond, A., \& Kirkham, N. Z. (2005). Not quite as grown-up as we like to think. parallels between cognition in childhood and adulthood. Psychological Science, 16(4), 291-297.

Dibbets, P., \& Jolles, J. (2006). The switch task for children: Measuring mental flexibility in young children. Cognitive Development, 21(1), 60.

Ellefson, M. R., Shapiro, L. R., \& Chater, N. (2006). Asymmetrical switch costs in children. Cognitive Development, 21(2), 108.

Garner, W. R. (1970). The stimulus in information processing. American Psychologist, 25(4), 350358.

Garner, W. R., \& Felfoldy, G. L. (1970). Integrality of stimulus dimensions in various types of information processing. Cognitive Psychology, 1(3), 225-241.

Hale, S. (1990). A global developmental trend in cognitive processing speed. Child Development, 61(3), 653-663.
Huizinga, M., Dolan, C. V., \& van der Molen, M. W. (2006). Age-related change in executive function: Developmental trends and a latent variable analysis. Neuropsychologia, 44(11), 2017.

Kail, R. (1986). Sources of age differences in speed of processing. Child Development, 57(4), 969987.

Kail, R. (1991). Developmental change in speed of processing during childhood and adolescence. Psychological Bulletin, 109(3), 490.

Kirkham, N. Z., Cruess, L., \& Diamond, A. (2003). Helping children apply their knowledge to their behavior on a dimension-switching task. Developmental Science, 6(5), 449-476

Kirkham, N. Z., \& Diamond, A. (2003). Sorting between theories of perseveration: performance in conflict tasks requires memory, attention and inhibition. Developmental Science, 6(5), 474476

Kloo, D., \& Perner, J. (2005). Disentangling dimensions in the dimensional change cardsorting task. Developmental Science, 8(1), 4456.

Kloo, D., Perner, J., Kerschhuber, A., Dabernig, S., \& Aichhorn, M. (2008). Sorting between dimensions: Conditions of cognitive flexibility in preschoolers. Journal of Experimental Child Psychology, 100(2), 115-134.

Koch, I., \& Allport, A. (2006). Cue-based preparation and stimulus-based priming of tasks in task switching. Memory \& Cognition, 34(2), 433-444.

Kray, J., Eber, J., \& Lindenberger, U. (2004). Age differences in executive functioning across the lifespan: The role of verbalization in task preparation. Acta Psychologica, 115(2-3), 143165.

Kray, J., \& Eppinger, B. (2006). Effects of associative learning on age differences in taskset switching. Acta Psychologica, 123(3), 187.

Kray, J., Li, K. Z. H., \& Lindenberger, U. (2002). Age-related changes in task-switching components: The role of task uncertainty. Brain and Cognition, 49, 363-381.

Kray, J., \& Lindenberger, U. (2000). Adult age differences in task switching. Psychology and Aging, 15, 126-147.

Liston, C., Matalon, S., Hare, T. A., Davidson, M. C., \& Casey, B. J. (2006). Anterior Cingulate and Posterior Parietal Cortices Are Sensitive to Dissociable Forms of Conflict in a TaskSwitching Paradigm. Neuron, 50(4), 643-653.

Mayr, U. (2001). Age differences in the selection of mental sets: The role of inhibition, stimulus ambiguity, and response-set overlap. Psychology and Aging, 16(1), 96-109.

Mayr, U., \& Bryck, R. L. (2007). Outsourcing control to the environment: effects of 
stimulus/response locations on task selection. Psychological Research, 71(1), 107-116.

Meiran, N. (1996). Reconfiguration of processing mode prior to task performance. Journal of Experimental Psychology: Learning, Memory, and Cognition, 22(6), 1423-1442.

Meiran, N. (2000). Reconfiguration of stimulus task sets and response task sets during task switching. In S. Monsell \& J. S. Driver (Eds.), Control of cognitive processes: Attention and performance XVIII (pp. 377-399). Cambridge, MA: MIT Press.

Meiran, N., Chorev, Z., \& Sapir, A. (2000). Component processes in task switching. Cognitive Psychology, 41(3), 211-253.

Monsell, S. (2003). Task switching. Trends in Cognitive Sciences, 7(3), 134-140.

Monsell, S., Yeung, N., \& Azuma, R. (2000). Reconfiguration of task-set: Is it easier to switch to the weaker task? Psychological Research, 63(3), 250-264.

Perner, J., \& Lang, B. (2002). What causes 3-yearolds' difficulty on the dimensional change card sorting task? Infant and Child Development, 11(2), 93-105.

Reimers, S., \& Maylor, E. A. (2005). Task switching across the life span: Effects of age on general and specific switch costs. Developmental Psychology, 41(4), 661.

Rennie, D. A. C., Bull, R., \& Diamond, A. (2004). Executive Functioning in Preschoolers: Reducing the Inhibitory Demands of the Dimensional Change Card Sort Task. Developmental Neuropsychology, 26(1), 423443.

Ridderinkhof, K. R., van der Molen, M. W., Band, G. P. H., \& Bashore, T. R. (1997). Sources of interference from irrelevant information: A developmental study. Journal of Experimental Child Psychology, 65(3), 315-341

Rogers, R. D., \& Monsell, S. (1995). Costs of a predictible switch between simple cognitive tasks. Journal of Experimental Psychology: General, 124(2), 207-231.

Rubin, O., \& Meiran, N. (2005). On the origins of the task mixing cost in the cuing task-switching paradigm. Journal of Experimental Psychology-Learning Memory and Cognition, 31(6), 1477-1491.

Schouten, A., Oostrom, K. J., Peters, A. C. B., Verloop, D., \& Jennekens-Schinkel, A. (2000). Set-shifting in healthy children and in children with idiopathic or cryptogenic epilepsy. Developmental Medicine and Child Neurology, 42(6), 392-397

Shepp, B. E., \& Barrett, S. E. (1991). The development of perceived structure and attention: Evidence from divided and selective attention tasks. Journal of Experimental Child Psychology, 51(3), 434.
Sohn, M., \& Anderson, J. R. (2003). Stimulusrelated priming during task switching. Memory \& Cognition, 31(5), 775-780.

Strutt, G. F., Anderson, D. R., \& Well, A. D. (1975). A developmental study of the effects of irrelevant information on speeded classification. Journal of Experimental Child Psychology, 20(1), 127-135.

Towse, J. N., Redbond, J., Houston-Price, C. M. T., \& Cook, S. (2000). Understanding the dimensional change card sort: Perspectives from task success and failure. Cognitive Development, 15(3), 347.

Waszak, F., Hommel, B., \& Allport, A. (2003). Task-switching and long-term priming: Role of episodic stimulus-task bindings in task-shift costs. Cognitive Psychology, 46, 361-413.

Welsh, M. C., Pennington, B. F., \& Groisser, D. B. (1991). A normative-developmental study of executive function: A window on prefrontal function in children. Developmental Neuropsychology, 7(2), 131-149.

Wylie, G., \& Allport, A. (2000). Task switching and the measurement of "switch costs". Psychological Research, 63(3 - 4), 212.

Yeung, N., \& Monsell, S. (2003a). Switching between tasks of unequal familiarity: The role of stimulus-attribute and response-set selection. Journal of Experimental Psychology: Human Perception and Performance, 29(2), 455-469.

Yeung, N., \& Monsell, S. (2003b). The effects of recent practice on task switching. Journal of Experimental Psychology: Human Perception and Performance, 29(5), 919-936.

Zelazo, P. D., Frye, D., \& Rapus, T. (1996). An age-related dissociation between knowing rules and using them. Cognitive Development, 11(1), 37-63.

Zelazo, P. D., Muller, U., Frye, D., \& Marcovitch, S. (2003). The development of executive function in early childhood. Monographs of the Society for Research in Child Development, $68(3)$ 